\title{
Radiation Effects on YAG:Ce Scintillating Fiber
}

\author{
Briana J. Singleton, Bradley S. Jones, Abigail A. Bickley, James C. Petrosky, Member, IEEE, John W. McClory, \\ Member, IEEE, Benjamin R. Kowash, Member, IEEE
}

\begin{abstract}
The performance of thin, cerium activated, yttrium aluminum garnet (YAG:Ce), scintillating fiber-shaped crystals were investigated following ion irradiation. The optical behavior of the YAG:Ce fibers was simulated using DETECT2000, a Monte Carlo based software program designed to model the optical properties of scintillators. The fibers were exposed to a focused beam of 0.5 and $1 \mathrm{MeV}$ electrons. Fiber light output and color shift were analyzed following irradiation.
\end{abstract}

\section{INTRODUCTION}

$\mathrm{T}$ HIN, inorganic YAG:Ce scintillating fibers provide a unique medium and output for radiation detection in harsh environments, such as space. These fibers are very stable, fast, and offer superior energy resolution over other brighter scintillators, but suffer from low active volume due to $\mathrm{Ce}$ oxidation during production [1]. Thus, quality light transport is of substantial interest in use of such fibers. Recent research investigated the properties of YAG:Ce fibers when coupled to silicon photomultipliers (SiPMs) for use in particle tracking and calorimetric applications [2].

SiPMs are relatively new, efficient, single photon sensitive, solid-state photodiode arrays which are well suited for the readout of scintillating fibers. A SiPM manufactured by Hamamatsu, the Multi-Pixel Photon Counter (MPPC) was used to measure the scintillation decay time profiles of six 400 $\mu \mathrm{m}$ YAG:Ce fiber crystals, grown by the Fibercryst Company, under alpha and gamma irradiation. Prior to conducting any experiments with the YAG:Ce fibers, the response of the MPPC was independently tested by measuring the time domain fluorescence decay profile and pulse height spectrum from another common scintillator material. A $1 \times 1 \times 12 \mathrm{~mm}^{3}$ cerium activated lutetium-yttrium oxyorthosilicate (LYSO:Ce) crystal was coupled to the MPPC using BC-630 ( $\mathrm{n}=1.46)$ optical grease. LYSO:Ce is a bright $(\sim 28,000 \mathrm{ph} / \mathrm{MeV})$ and fast scintillator which, unlike YAG:Ce, has only one fast component around 42 ns [3]. Additionally, LYSO:Ce has the same refractive index as YAG:Ce $(n=1.82)$. The LYSO:Ce sample used in this experiment was grown via the $\mu$-PD method by Fibercryst.

The scintillation response of the LYSO:Ce to gamma radiation from a ${ }^{22} \mathrm{Na}$ point source was measured directly from the

Manuscript received November 22, 2011

B. J. Singleton is with the Air Force Institute of Technology, WrightPatterson AFB, OH 45433 USA (telephone: 937-255-3636, e-mail: briana.singleton@afit.edu).

B. S. Jones, was with Air Force Institute of Technology, Wright-Patterson, OH 45433 USA. He is now with the $498^{\text {th }}$ Nuclear Systems Wing, Kirtland AFB, NM 87117 USA (telephone: 505-846-2009, e-mail: brad.jones@kirtland.af.mil).

The remaining authors are with the Air Force Institute of Technology, Wright-Patterson, OH 45433 USA, (telephone: 937-255-3636, e-mail: first.last@afit.edu).
MPPC with an oscilloscope. The time domain fluorescence decay measurements of LYSO:Ce were used to confirm proper MPPC response to a scintillation pulse. A typical pulse is shown below in Fig. 1. One thousand of the highest amplitude scintillation pulse waveforms that were digitized and collected were analyzed and confirmed the proper time domain response of the MPPC to LYSO:Ce scintillation.

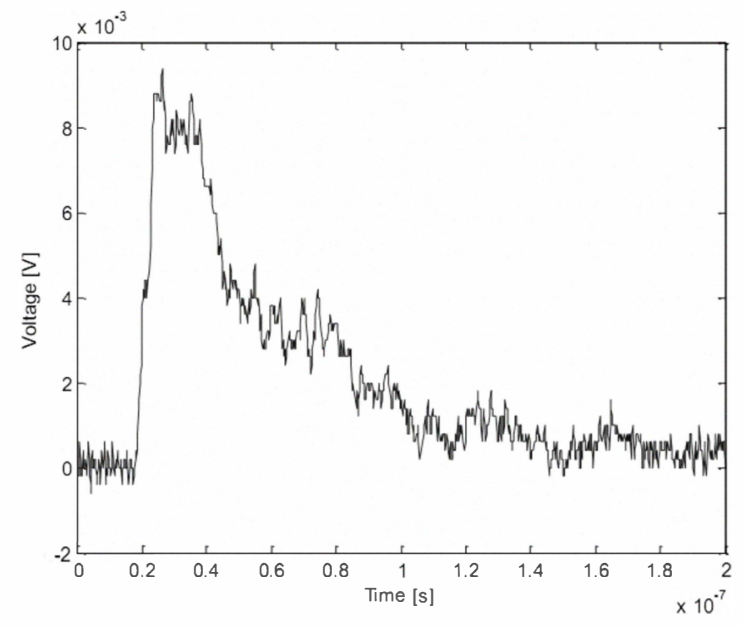

Figure 1 A typical digitized LYSO:Ce gamma scintillation pulse as measured directly using the MPPC readout circuit and $1 \mathrm{GSa}$ /s oscilloscope is shown.

Interestingly, the observed decay times in the thin YAG:Ce fibers were substantially shorter than values for bulk single crystal YAG:Ce reported in open scientific literature [4]. Additionally, both gamma and alpha scintillation measurements were conducted to estimate the energy resolution of the YAG:Ce fibers. The research highlighted the need to further investigate the radiation response of YAG:Ce fibers and to correlate the physical data with a sufficient model. The fibers were further investigated for sensitivity to point defects resulting from high-energy electron irradiation.

\section{EXPERIMENT}

Six YAG:Ce crystal fibers were used for the first set of experiments. The initial YAG:Ce experiment characterized the time domain fluorescence decay profile and amplitude of scintillation pulses from each of the six fibers measured directly from the MPPC readout. A bare ${ }^{241} \mathrm{Am}$ source was placed $5.8 \mathrm{~mm}$ directly underneath the test fiber, which is well below the mean range of $4 \mathrm{~cm}$ for the ${ }^{241} \mathrm{Am}$ alpha particle.

For each of the six fibers under study, 1600 alpha particle scintillation pulses were collected via MPPCs, directly digitized by the oscilloscope. Additionally, digitized scintillation waveforms for one fiber, F6, were collected during gamma irradiation from a bare ${ }^{22} \mathrm{Na}$ source. This fiber was wrapped in several layers of Teflon tape. These waveforms were digitally processed to compare the 
fluorescence decay profiles to the values published in literature.

The YAG:Ce decay profile fiber experiment was modeled in DETECT2000. Scintillation parameters from Ludziejewski, et al., were chosen for this model [4]. Specifically, a light yield of $17,000 \mathrm{ph} / \mathrm{MeV}$ was selected and an $\alpha / \gamma$ ratio of $21 \%$ was applied; therefore, the $5.4 \mathrm{MeV} \alpha$-particle was assumed to produce 17,000 scintillation photons (approximately accounting for energy lost due to particle straggling). Two decay constants, 88.9 ns and $458.5 \mathrm{~ns}$, were chosen with two independent intensities, $14.9 \%$ and $85.1 \%$ respectively. The refractive index of the $400 \mu \mathrm{m}$ diameter by $100 \mathrm{~mm}$ long cylindrical YAG:Ce fiber was set to 1.82 and the emission wavelength was set to $550 \mathrm{~nm}$, the emission peak of YAG:Ce. Air was used as the fiber cladding $(n=1.00)$. Importantly, the surface treatments were modeled as polished; this surface model applies only refraction and reflection due to changes in the refractive index between the air and the fiber. The two fiber ends both had $100 \%$ efficient detectors with a thin coating of index matching fluid $(n=1.46)$. The data from DETECT2000 was processed and convolved with the MPPC single pixel response model to produce a fully simulated YAG:Ce $5.4 \mathrm{MeV}$ alpha particle scintillation pulse as measured via the readout electronics.

Energy calibration measurements were taken using the YAG:Ce fibers coupled to a single fiber end MPPC readout and compared to a single fiber end PMT readout. For the MPPC readout experiments, an ${ }^{241} \mathrm{Am}$ alpha source was collimated using a $4.7 \mathrm{~mm}(3 / 16$ ") thick circular aluminum plate with a $1 \mathrm{~mm}$ diameter hole drilled out. The alpha source was placed $5.8 \mathrm{~mm}$ directly underneath the center of the mounted YAG:Ce fiber. In this configuration, the spread of alpha particles along the length of the fiber is limited to 2.4 $\mathrm{mm}$. This experiment was setup to minimize variation in the amount of scintillation light produced and detected in the YAG:Ce fiber.

In addition to alpha radiation, several gamma sources were also collimated and used to irradiate the YAG:Ce fibers during MPPC readout. Again, because of the low count rates from gamma irradiation of YAG:Ce, the energy resolution measurements from a ${ }^{22} \mathrm{Na}$ source were taken using only the one Teflon-wrapped fiber, F6. These sources were placed behind a $2.54 \mathrm{~cm}$ thick lead brick with a $1 \mathrm{~mm}$ diameter hole removed for collimation. The resulting spread of the gamma beam at a distance of $7.52 \mathrm{~cm}$ is only $6 \mathrm{~mm}$.

The YAG:Ce energy resolution measurements were made using the photodetector (i.e. either MPPC or PMT) connected in series to a Cremat CR111 charge integrating preamplifier, an Ortec 672 shaping amplifier with a $1 \mu$ s shaping time, and an ADCAM multi-channel analyzer.

Finally, nine fiber pieces were selected and affixed to a copper cold head and irradiated with electrons in the Dynamitron operated at Wright-State University, OH. A spectrometer with CCD sensor was used to view the spectral output of the fibers during irradiation. The fibers were irradiated for approximately $1.5 \mathrm{hrs}$ using electron energies of $500 \mathrm{keV}$ and $1 \mathrm{MeV}$ and current from 0.5 to $2 \mu \mathrm{A}$.

\section{RESULTS}

\section{A. Decay Profile}

The scintillation pulse shapes of 1600 events were recorded, summed together (i.e. filtering the MPPC signal), and normalized for each fiber, the results are shown in Fig. 2.

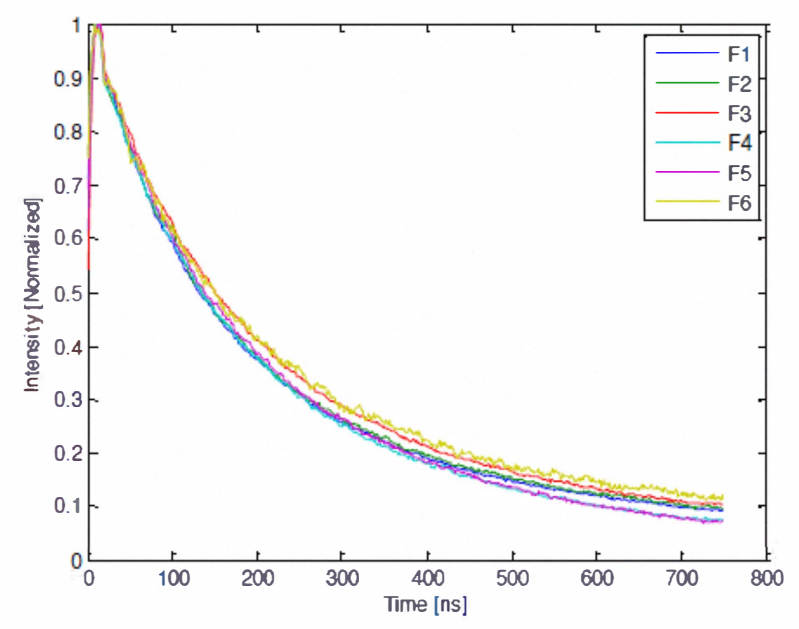

Figure 2 The fluorescence decay profiles of six YAG:Ce fibers are shown after summing and normalizing over hundreds of scintillation pulses. The tails show slightly differing decay times.

The decay profile for each fiber was fit using a sum two exponentials for each fiber and the fit parameters are given in Table 1 . The fit began at the peak of the scintillation pulse (nominally $10 \mathrm{~ns}$ ) and concluded at $750 \mathrm{~ns}$. For comparison, data from [4] and [5] indicates that for bulk crystals, the fast component of YAG:Ce scintillation increased from $\sim 105$ to $115 \mathrm{~ns}$ under gamma irradiation to around 68 to $89 \mathrm{~ns}$ under alpha irradiation, increasing with cerium activator concentration.

TABle 1 Alpha Particle Scintillation Properties of YAG:Ce Fibers

\begin{tabular}{|c|c|c|c|c|c|c|c|c|}
\hline \multirow{2}{*}{$\begin{array}{c}\text { Fiber } \\
{[\#]}\end{array}$} & \multicolumn{2}{|c|}{ Fast Component } & \multicolumn{2}{|c|}{ Total Intensity - Fast } & \multicolumn{2}{|c|}{ Slow Component } & \multicolumn{2}{|c|}{ Total Intensity - Slow } \\
\hline & {$[\mathrm{ns}]$} & $\pm \mathrm{ns}$ & {$[\%]$} & $\lfloor \%$ & {$[\mathrm{ns}]$} & $\pm \mathrm{ns}$ & {$[\%]$} & $\pm \%$ \\
\hline 1 & 115.27 & 1.13 & $28.39 \%$ & $0.28 \%$ & 519.75 & 7.12 & $71.61 \%$ & $1.00 \%$ \\
\hline 2 & 125.08 & 1.43 & $30.77 \%$ & $0.36 \%$ & 588.93 & 12.28 & $69.23 \%$ & $1.48 \%$ \\
\hline 3 & 139.61 & 1.63 & $31.77 \%$ & $0.37 \%$ & 587.20 & 12.45 & $68.23 \%$ & $1.48 \%$ \\
\hline 4 & 126.33 & 1.71 & $31.85 \%$ & $0.44 \%$ & 436.68 & 7.53 & $68.15 \%$ & $1.21 \%$ \\
\hline 5 & 113.69 & 1.89 & $24.65 \%$ & $0.42 \%$ & 373.27 & 4.77 & $75.35 \%$ & $0.98 \%$ \\
\hline 6 & 122.52 & 2.41 & $24.45 \%$ & $0.49 \%$ & 562.75 & 14.66 & $75.55 \%$ & $2.03 \%$ \\
\hline
\end{tabular}

As shown in Table 1, the fast component decay times and relative intensities of YAG:Ce fiber scintillation are in agreement. However, the fast components are significantly longer than the 68 to 89 ns scintillation decay of the bulk YAG:Ce as reported in [4] by Ludziejewski et al. in response to alpha radiation. In fact, the fast decay component of the alpha scintillation more closely matches $105-115$ ns decay associated with gamma scintillation as reported in [4]. The slow decay components of the alpha scintillation reasonably compare to the slow components measured by [4]. The trends 
of the relative intensities are also in agreement with the results reported by Moszynski et al. [5]. Of course, the materials studied by these groups are assumed to be homogeneously doped with a cerium activator. This is unlikely the case for the single crystal fibers grown via $\mu-P D$, as shown in [6], [7], and [8].

The fast and slow decay components of $\mathrm{F} 6$ in response to gamma scintillation are $128.1 \pm 2.4 \mathrm{~ns}$ and $561.8 \pm 56.8 \mathrm{~ns}$ with respective intensities of $56.6 \pm 1.1 \%$ and $43.4 \pm 4.8 \%$. Interestingly, as shown in Fig. 3, the decay time constants are nearly identical to the fiber's alpha scintillation response. Thus, the decay time constant change between alpha and gamma scintillation in bulk YAG:Ce observed by Moszynski et al. is not apparent in this fiber shaped sample.

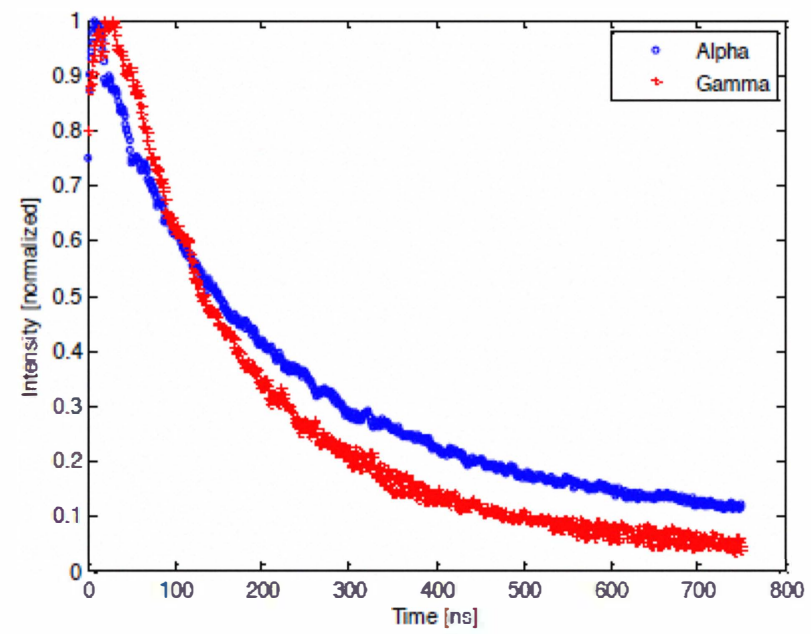

Figure 3 The fluorescence decay profile for fiber F6 under gamma excitation is shown compared to alpha scintillation decay profile. Interestingly, the fast and slow decay constants are nearly identical for alpha and gamma excitation, but the intensities flip.

The readout of alpha scintillation waveforms from the YAG:Ce fibers using MPPCs was simulated in DETECT2000. The fully simulated $5.4 \mathrm{MeV}$ alpha scintillation MPPC waveform is shown compared to the mean of 8,000 measured alpha scintillation waveforms in Fig. 4. The overall signal amplitude of the measured alpha scintillation waveforms are in good agreement with the results of the simulation. In general, fewer pixels fired in measurement than in simulation which may indicate a lower than expected scintillation yield. This could be explained by particle straggling, or perhaps by a lower concentration of Ce activators which is supported by the longer than expected scintillation decay times.

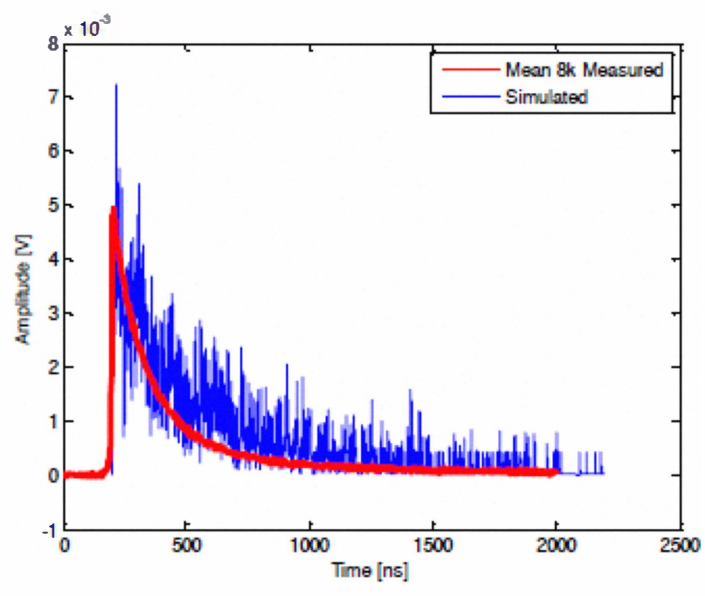

Figure 4 A fully simulated alpha scintillation waveform in a YAG:Ce fiber as read out using a MPPC is compared to the mean of 8,000 measured waveforms

\section{B. Energy Spectrum and Resolution}

Attempts to measure the achievable energy resolution using YAG:Ce fibers with fiber end MPPC readout were unsuccessful due to the lack of a measureable photopeak using gamma sources. Spectra were collected using traditional NIM spectroscopy electronics (e.g. charge integrating preamplifier, shaping amplifier, and multi-channel analyzer). A typical measured spectrum is shown in Fig. 5, taken under gamma irradiation from $\mathrm{a}^{22} \mathrm{Na}$ source.

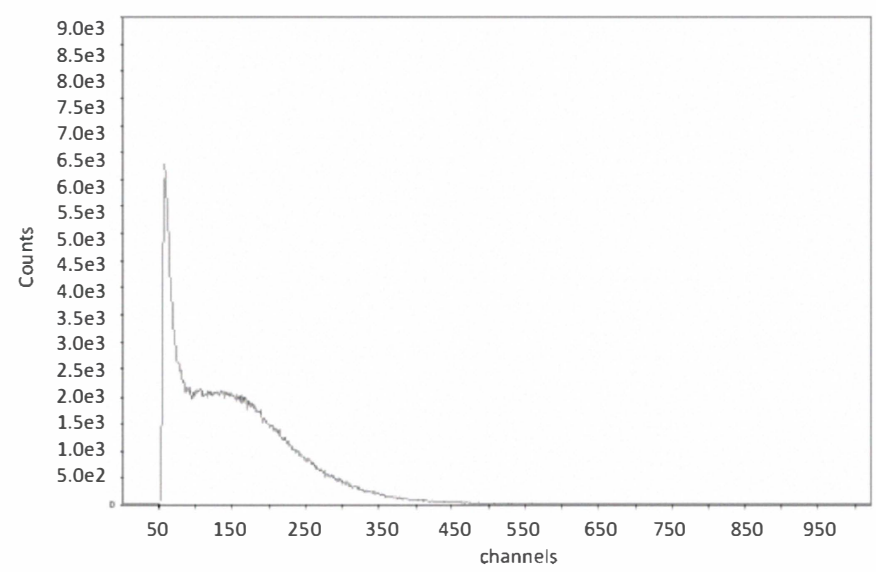

Figure $5{ }^{22} \mathrm{Na}$ gamma energy spectrum using MPPC readout, NIM spectroscopy electronics, and a YAG:Ce scintillating fiber. Noise integration is apparent at around channel 75 indicating very noisy electronics. A feature resembling a Compton edge appears around channel 200.

Extremely low gamma efficiencies should be expected from such thin scintillating crystals, especially those with low stopping power such as YAG:Ce. Consequently, alpha spectroscopy is better suited. The spectrum of an ${ }^{241} \mathrm{Am}$ source using the same fiber and electronic settings is shown in Fig. 6.

The Compton edge of a $511 \mathrm{keV}$ gamma ray lies at 340 $\mathrm{keV}$. If one accepts that the Compton edge lies near channel $\# 200$ of Fig. 5 and assumes an $\alpha / \gamma$ ratio of $21 \%$ in YAG:Ce, then the peak of the $5.4 \mathrm{MeV}$ alpha associated with ${ }^{241} \mathrm{Am}$ should be expected at channel \#667, which agrees with the data in Fig. 6. These results are no substitute for a proper 
energy calibration, to say the least; rather, they merely serve as sanity check to the system response.

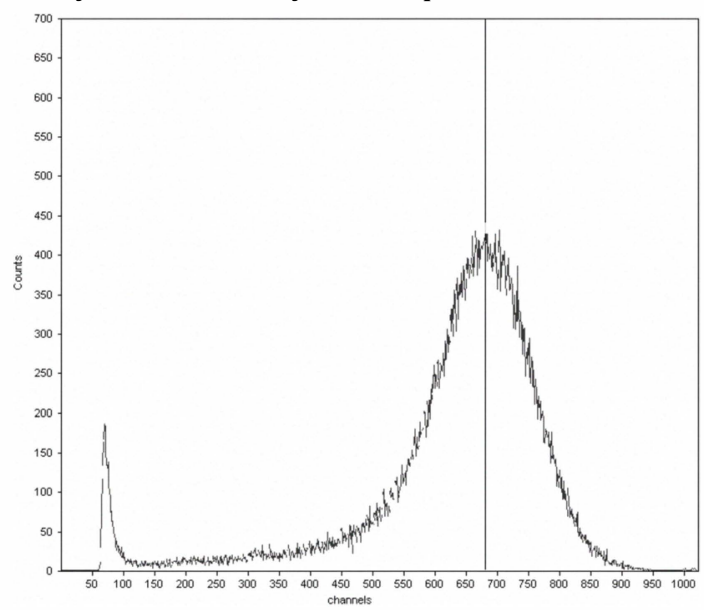

Figure $6{ }^{241} \mathrm{Am}$ alpha spectrum shown using the same readout and settings as the ${ }^{22} \mathrm{Na}$ spectrum in Fig. 51. Noise integration is apparent centered at channel 75 indicating very noisy electronics. The alpha peak is centered around channel \#667.

The results of the alpha spectroscopy experiment are expected. The spectrum shown in Fig 6 represents a typical alpha spectrum; there is a well defined (electronic noise broadened) peak with a low energy tail which represents the particles that lost energy along their tracks to the detector (i.e. straggling). However, the gamma energy spectrum lacks distinguishing spectral features making it difficult to calibrate and impossible to determine energy resolution.

To explain the lack of resolution, the experiment was simulated using a popular particle tracking code called Monte Carlo N-Particle transport code, version 5 (MCNP5) [9]. The simulated energy spectra of a pure YAG fiber from several monoenergetic gamma ray sources at two angles of incidence indicate that the YAG:Ce fibers are inherently inefficient by themselves and have extremely low photofractions (i.e. the ratio of the area under the photopeak to the entire response function). The $511 \mathrm{keV}$ photofraction is about $3.5 \%$ while the $2.6 \mathrm{MeV}$ photofraction is only $0.2 \%$. The spectrum measured in the lab had fewer overall counts and it is therefore unreasonable to expect a distinct photopeak. The low count rate is also a result of the experimental setup where the fibers were illuminated from the side. Based upon the MCNP5 simulations, the fibers are more efficient when illuminated along the fiber axis.

In addition to inefficiency, the distribution of the $\mathrm{Ce}$ activator in the fiber may have a significant impact on the poor energy resolution. The Ce concentration of YAG:Ce grown via $\mu$-PD has strong radial dispersion. In fact, the fiber may be more closely modeled as a shell of highly doped YAG:Ce with a lightly doped core rather than a homogeneous material. As a result, when the Compton recoil electrons or photoelectrons deposit their energy along relatively long tracks, they excite charge carriers in both regions of high and low Ce concentration within the fiber crystal. Since the scintillation efficiency of the fiber crystal is dependent on the activator concentration, the total amount of light produced during scintillation is dependent on the location of interaction and the subsequent localized densities of charge carriers within fiber.

Although the absolute quantities of cerium activator could not be measured in the fibers via X-ray fluorescence microscopy or SEM due to low atomic concentrations (i.e. 0.1 atm.\%), clear radial dispersion of the cerium can be observed via UV fluorescence microscopy. A $100 \mu \mathrm{m}$ thick crosssectional slice of YAG:Ce fiber was cut by technicians at the Air Force Research Laboratory Materials and Manufacturing Directorate. The slice was then photographed under a UV microscope. As shown in Fig. 7, there is clearly a radial dispersion of activator as indicated by the increase in luminous intensity near the fiber rim.

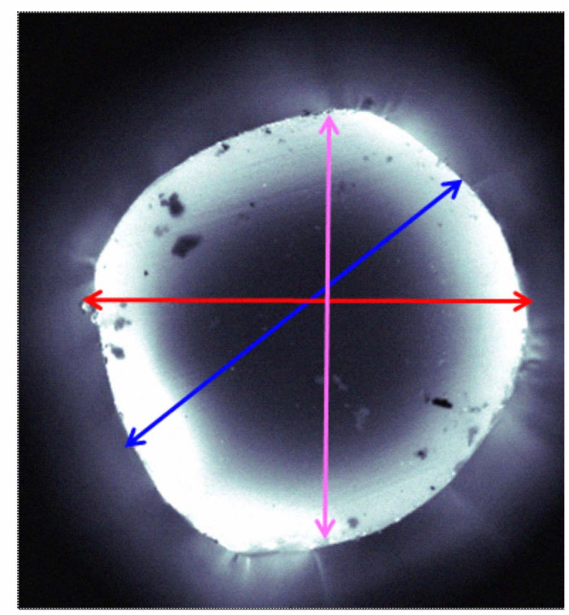

Figure 7 The green channel from an image of a YAG:Ce fiber cross-section under UV fluorescence

The intensity of the green channel was plotted in Fig 8 for three vectors across the fiber, as shown in Fig. 7. However, the camera's 8-bit ADC was saturated by the intense luminescence at even the lowest UV illumination setting; this is shown by the plateau at values of 255 in Fig. 8.

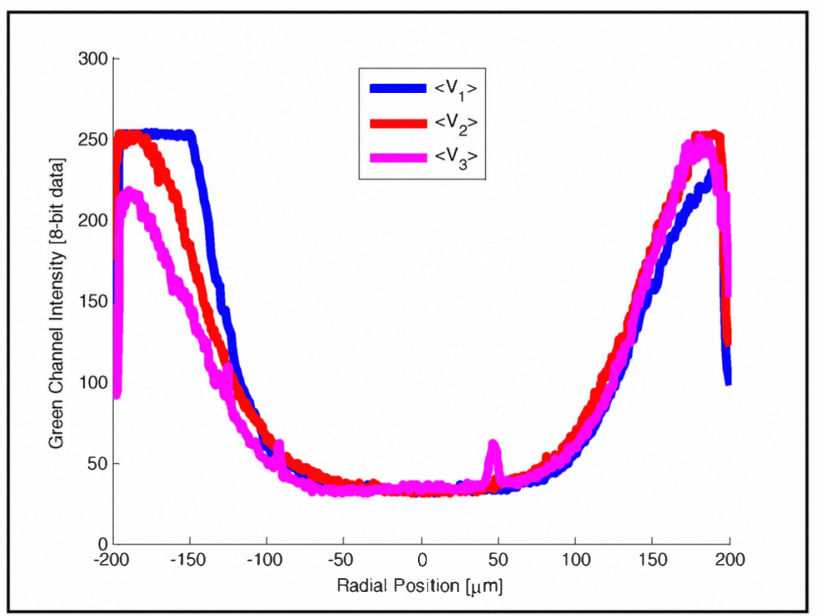

Figure 8 The green channel intensity is shown as a function of radial position for the three different diameter vectors shown in blue, red, and magenta in Fig. 7. Notice that the CCD's 8-bit ADC is saturated even at the lowest UV illumination setting. 
Although this data does not indicate the cerium concentration directly, one might assume a linear relationship between the concentration and light yield, as shown for LuAG:Ce by Dujardin, et al., in [8].

\section{Electron Irradiation}

The light output from nine pieces of YAG:Ce crystal fiber was monitored during room temperature electron irradiation in a Dynamitron. The two peaks associated with the splitting of the Ce ground state into two levels in YAG crystal are clearly seen in the spectrum, Fig. 9 at approximately 526 and $572 \mathrm{~nm}$ [10]. The scintillation response of the fibers is linear at the 500 $\mathrm{keV}$ electron energy as seen in the relative increases in Fig. 9.

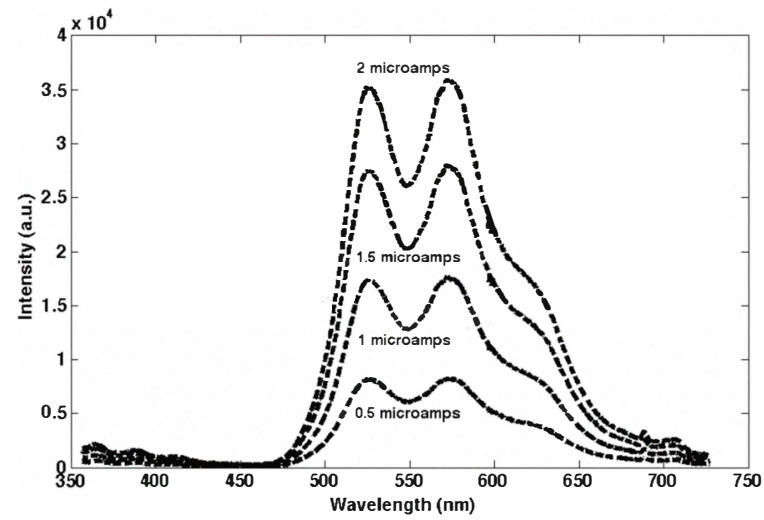

Figure 9 Spectral response of YAG:Ce fibers to $500 \mathrm{keV}$ electrons at various current levels. The fibers demonstrate a linear response to flux at this electron energy.

At $1 \mathrm{MeV}$, the response is not as what is measured for 500 $\mathrm{keV}$ electrons. The magnitude of the intensity is diminished due to the reduced interaction of the higher energy electrons within the YAG:Ce fibers. This is not unexpected as the Bethe-Bloch formulation, plotted in Fig. 10 for the total stopping power for electrons in YAG:Ce actually reaches a minimum near $1 \mathrm{MeV}$ [11].

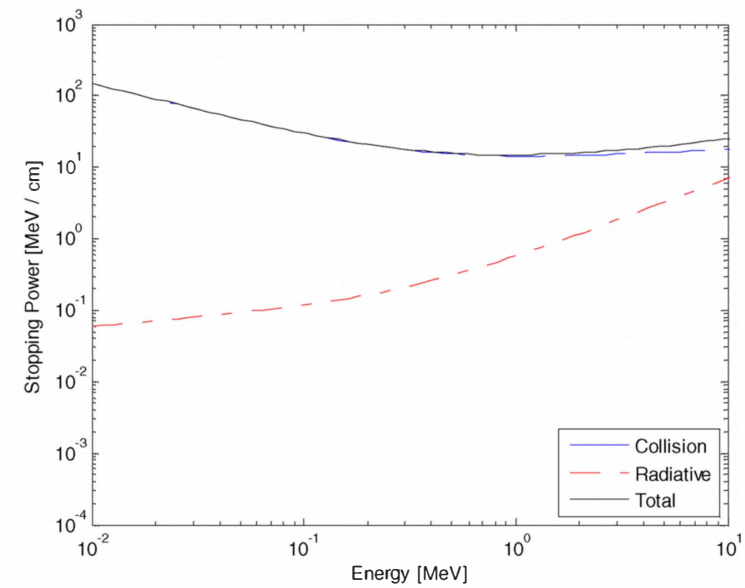

Figure 10 The fast electron stopping powers in YAG:Ce calculated using the Bethe-Bloch formula is plotted. The total stopping power is at a minimum near $1 \mathrm{MeV}$.
A curve fitting routine was used to identify the peaks in the spectrum. The intensity of the peaks at the various currents level for $500 \mathrm{keV}$ and $1 \mathrm{MeV}$ electrons is plotted in Fig. 11.

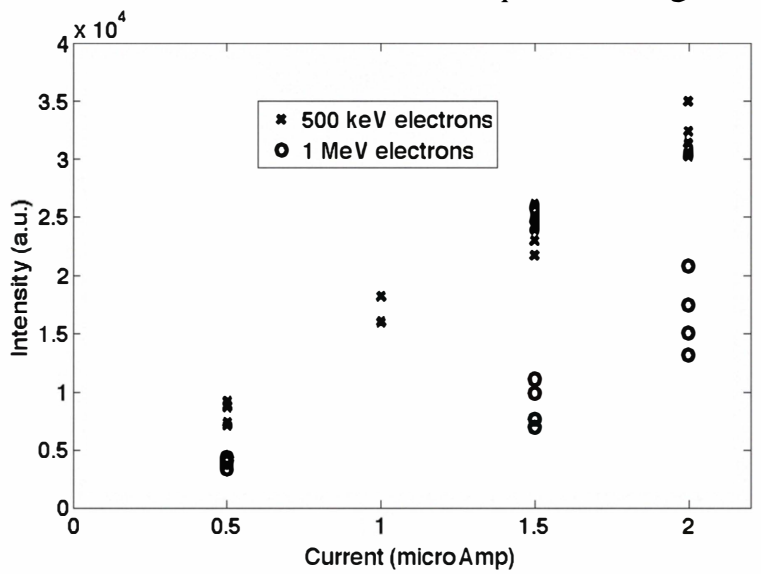

Figure 11 Peak intensity as a function of current. At the $500 \mathrm{keV}$ electron energy, the peak intensity increases linearly with increasing current. At 1 $\mathrm{MeV}$, the response is not super-linear.

The identified peaks are plotted as a function of irradiation time in Fig. 12. The fibers were irradiated by $500 \mathrm{keV}$ electrons up to $62 \mathrm{~min}$. The fibers were then irradiated for 24 min with $1 \mathrm{MeV}$ electrons. From Fig. 12, an upward trend to the peak wavelength is visible, but it is within the error of the data.
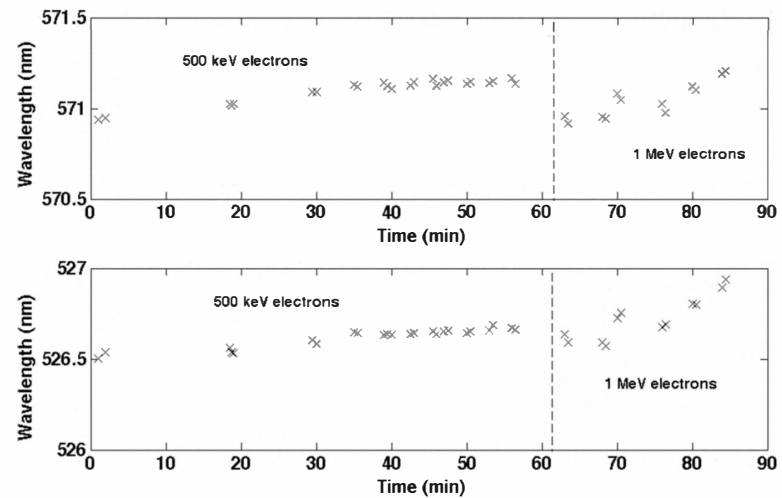

Figure 12 The wavelength of the peak is plotted as a function of irradiation time.

\section{CONCLUSION}

The ability to calorimetrically measure and track gamma ray interactions in bundles of scintillating fibers has important applications in numerous fields ranging from medical imaging to national security. Compton imaging of SNM at standoff ranges is one such application. This research focused on predicting, modeling, and measuring the achievable energy and position resolutions in YAG:Ce fiber crystals using SiPMs for such an application.

Inorganic fiber scintillators coupled to highly efficient SiPMs is a promising new concept for a gamma ray detection system. Unfortunately, due to the inefficiency (i.e. low density, low effective Z) of the material and its small volume, no energy resolution could be measured using a single $400 \mu \mathrm{m}$ YAG:Ce fiber with dual end MPPC readout. Radial dispersion of cerium activator concentration caused by growth 
via $\mu$-PD is suspected to cause smearing of high gamma interactions due to the large range of recoil and photoelectrons in YAG:Ce.

Alpha and gamma radiation of YAG:Ce fibers showed different scintillation decay times, as reported in literature. However, the fast and slow components did not change substantially as observed by Ludziejewski, et al., and Moszynski, et al.; instead, only a change in relative decay component intensities was observed between alpha and gamma irradiation. Additionally, the alpha scintillation fast component was consistently measured above $113 \mathrm{~ns}$ in all fiber samples, much slower than reported values (e.g. 68 to 89 ns, depending on cerium concentration) for bulk YAG:Ce.

Spectral output of the fibers under exposure to high-energy electrons was measured for $500 \mathrm{keV}$ and $1 \mathrm{MeV}$ electrons. The output of the fibers showed a linear response to flux at $500 \mathrm{keV}$, however the response at $1 \mathrm{MeV}$ fit best to a quadratic curve. Further work to investigate this phenomenon will be conducted.

\section{REFERENCES}

[1] Philippe Anfre, et al., "Evaluation of fiber-shaped LYSO for double readout gamma photon detection," IEEE Trans. on Nucl. Sci., vol. 54, no. 2, pp. 391-397, April 2007

[2] Jones, Bradley S., Investigation of YAG:Ce Scintillating Fiber Properties Using Silicon Photomultipliers, MS Thesis. Air Force Institute of Technology. 2011.

[3] L. Pidol, et al., "High efficiency of lutetium silicate scintillators, Cedoped LPS, and LYSO crystals," IEEE Trans. on Nucl. Sci., vol. 51, no. 3, pp. 1084-1087, June 2004

[4] T. Ludziejewski, et al., "Investigation of some scintillation properties of YAG:Ce crystals," Nuclear Instruments and Methods, vol. A 398, pp. 287-294, 1997.

[5] M. Moszynski and et al., "Properties of the YAG:Ce scintillator," Nuclear Instruments and Methods, vol. A 345, pp. 461-467, 1994.

[6] Philippe Anfré, "Localisation des interactions de rayonnements ionisants sur des fibres monocristallines scintillatrices," University of Lyon, Lyon, France, PhD Thesis 2007.

[7] Tsuguo Fukada and Valery I. Chani, Shaped Crystals: Growth by MicroPulling-Down Technique. New York, USA: Springer Berlin Heidelberg, 2007.

[8] C. Dujardin and et al., "LuAG:Ce fibers for high energy calorimetry," Journal of Applied Physics, vol. 108, pp. 013510:1-7, 2010.

[9] Monte Carlo Codes Group - Los Alamos National Laboratory. (2011, February) MCNP - A General Monte Carlo N-Particle Transport Code Version 5. [Online]. mcnp-green.lanl.gov

[10] R.R. Jacobs, W.F. Krupke, M.J. Weber, "Measurement of excited-stateabsorption loss for $\mathrm{Ce} 3+$ in $\mathrm{Y} 3 \mathrm{Al} 5 \mathrm{O} 12$ and implications for tunable $5 \mathrm{~d} \rightarrow 4$ f rare-earth lasers," Appl. Phys. Lett. 33 (1978) 410

[11] James E. Turner, Atoms, Radiation, and Radiation Protection, 2nd ed. New York City, USA: Wiley-Interscience, 1995. 